\title{
FRCR Part 1: Anatomy Mock Examinations
}

A. Shaw, B. Smith, and D.C. Howlett, eds.

New York, NY: Cambridge University Press, 2011, 228 pages, $\$ 42$

Examinations are an essential part of the education system and make an important contribution to the high standard of medicine in the United Kingdom. Structured testing is the objective and effective method of testing knowledge. It is possible to combine questions with images, thus bringing this form of testing closer to the reality of everyday life. In 2010 the Royal College of Radiologists reintroduced the anatomy component of the FRCR Part I examination, which is $75 \mathrm{~min}$ long and consists of 20 radiologic images with 5 questions on each.

This relatively small book provides essential practice for the new anatomy examinations introduced by the Royal College of Radiologists. It contains 10 mock examinations and 200 high-quality MRI, CT, ultrasonography, fluoroscopy, angiography, and plain film images. Nuclear medicine, including PET, is excluded. The book is broadly divided into 4 categories: head and neck, thorax, abdomen and pelvis, and musculoskeletal system. However, all anatomic areas are covered, including normal variants and pediatric cases. Each mock examination is laid out and structured in the same way as the actual test, ensuring that readers gain familiarity with both the content and the style. The answer sections include detailed explanations of the relevant anatomy, along with helpful learning tips and clinical applications.

The clear, succinct, readable questions of this outstanding book test important aspects of radiologic anatomy, and the excellent, high-quality images clearly demonstrate the findings. This book will prove popular with radiologists preparing for examinations. It also provides trainee radiologists with a focused and invaluable review, and it is complementary to existing anatomy textbooks. Its utility is not limited to radiologists; radiographers and medical students will also find the detailed images and descriptions helpful to their studies.

\author{
E. Edmund Kim \\ M.D. Anderson Cancer Center \\ 1400 Pressler, Unit 1483 \\ Houston, TX 77030 \\ E-mail: ekim@di.mdacc.tmc.edu
}

COPYRIGHT (c) 2012 by the Society of Nuclear Medicine and Molecular Imaging, Inc.

Published online Jun. 5, 2012.

DOI: 10.2967/jnumed.112.105445 